\title{
Do Magnetic Fields Actually Inflate Low-Mass Stars?
}

\author{
Gregory A. Feiden ${ }^{1,2}$ and Brian Chaboyer ${ }^{2}$ \\ ${ }^{1}$ Dept. of Physics \& Astronomy, Uppsala University, Box 516, Uppsala 751 20, Sweden. \\ ${ }^{2}$ Dept. of Physics \& Astronomy, Dartmouth College, 6127 Wilder Laboratory, Hanover, NH \\ 03755, USA. \\ email: gregory.a.feiden.gr@dartmouth.edu
}

\begin{abstract}
Magnetic fields have been hypothesized to inflate the radii of low-mass stars - defined as less than $0.8 M_{\odot}$-in detached eclipsing binaries (DEBs). We evaluate this hypothesis using the magnetic Dartmouth stellar evolution code. Results suggest that magnetic suppression of thermal convection can inflate low-mass stars that possess a radiative core and convective outer envelope. A scaling relation between X-ray luminosity and surface magnetic flux indicates that model surface magnetic field strength predictions are consistent with observations. This supports the notion that magnetic fields may be inflating these stars. However, magnetic models are unable to reproduce radii of fully convective stars in DEBs. Instead, we propose that model discrepancies below the fully convective boundary are related to metallicity.
\end{abstract}

Keywords. binaries: eclipsing, stars: evolution, stars: interiors, stars: low-mass, stars: magnetic field

\section{Introduction}

It has been well-documented over the past decade that stellar evolution models are unable to accurately predict radii and effective temperatures-so called "fundamental properties" - of low-mass stars $\left(M<0.8 M_{\odot}\right)$ in detached eclipsing binaries (DEBs; see, e.g., Ribas 2006, Feiden \& Chaboyer 2012a). Model radii have been shown to be too small and effective temperatures too hot, particularly in the most well-studied systems. Since stellar evolution models are heavily used to aid with the interpretation of observational data, it is crucial that these modeling errors be addressed. Magnetic fields, maintained by spin-orbit synchronization of DEB components, have been hypothesized to be the culprit. Magnetic activity indicators appear to correlate with radius discrepancies, supporting this hypothesis (e.g., López-Morales 2007). We aim to test this hypothesis using the recently developed magnetic Dartmouth stellar evolution code.

\section{Method}

We use models generated as a part of the Dartmouth Magnetic Evolutionary Stellar Tracks and Relations program (DMESTAR; Feiden \& Chaboyer 2012b, 2013a) to test whether model radii may be inflated by interactions between a magnetic field and thermal convection. Two techniques are used to incorporate magneto-convection in the models: (1) stabilization of convection, and (2) inhibition of convective efficiency (Feiden \& Chaboyer 2013a). Method one alters the Schwarzschild convective stability criterion by assuming the magnetic field is in equilibrium with the surrounding gas. An upper limit to the magnetic field strength occurs when the magnetic field is in thermal equipartition with the gas, approximately when the magnetic pressure is equal to the gas pressure. Method 

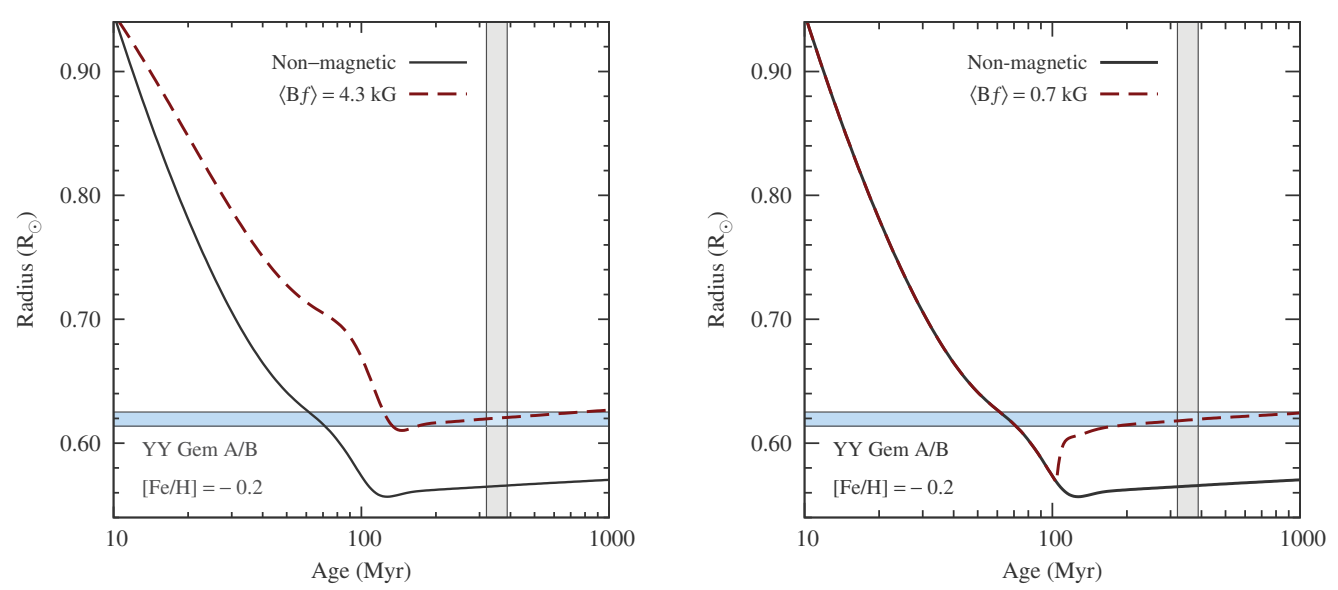

Figure 1. Radius evolution of standard (black, solid line) and magnetic (maroon, dashed line) models of the equal-mass DEB YY Gem. (Left) Magnetic model using stabilization of convection introduced at 10 Myr. (Right) Magnetic model invoking inhibition of convective efficiency initialized at $100 \mathrm{Myr}$. The perturbation age has no effect on results along the main sequence. The blue horizontal shaded region indicates the observed radius with associated $1 \sigma$ uncertainties and the vertical grey region highlights the estimated age of the system.

two instead assumes that the energy required to create the magnetic field is drawn from the kinetic energy of convective flows. Therefore the magnetic field strength has an upper limit determined by equipartition with convective flows, $B_{\text {eq }}=\left(4 \pi \rho u_{\text {conv }}^{2}\right)^{1 / 2}$.

Standard stellar evolution models (i.e., non-magnetic models) were run for both components of DEB systems to assess the level of radius inflation required of our magnetic models. A series of magnetic models were then computed with varying magnetic field strengths, using the magneto-convection techniques outlined above, until the model fundamental properties agreed with observationally determined values. We performed this procedure for several well-studied DEB systems to ascertain whether results were robust.

\section{Results}

Effects of magneto-convection on fundamental stellar properties predicted by stellar evolution models may be summarized for two different low-mass stellar populations: stars that have a radiative core with a convective outer envelope and fully convective stars. We will focus on model radius predictions, as stellar radii are more reliably measured from observations than effective temperatures.

The influence of magneto-convection on partially convective stars is demonstrated in Figure 1. The only significant difference between the two panels is the adopted magnetoconvection technique with stabilization of convection used in the left panel and inhibition of convective efficiency in the right panel. It is clear that accounting for magnetoconvection can inflate model radius predictions at a level required to reconcile models with observations. In general, we observe that both magneto-convection techniques provide a qualitatively correct solution for partially convective stars. However, the two magnetoconvection techniques predict significantly different surface magnetic field strengths. Inhibition of convective efficiency typically requires weaker surface magnetic field strengthsby roughly a factor of 5 - than stabilization of convection. For example, Figure 1 shows that stabilization of convection (left panel) requires a $4.3 \mathrm{kG}$ surface magnetic field while inhibition of convective efficiency (right panel) requires a $0.7 \mathrm{kG}$ surface magnetic field 

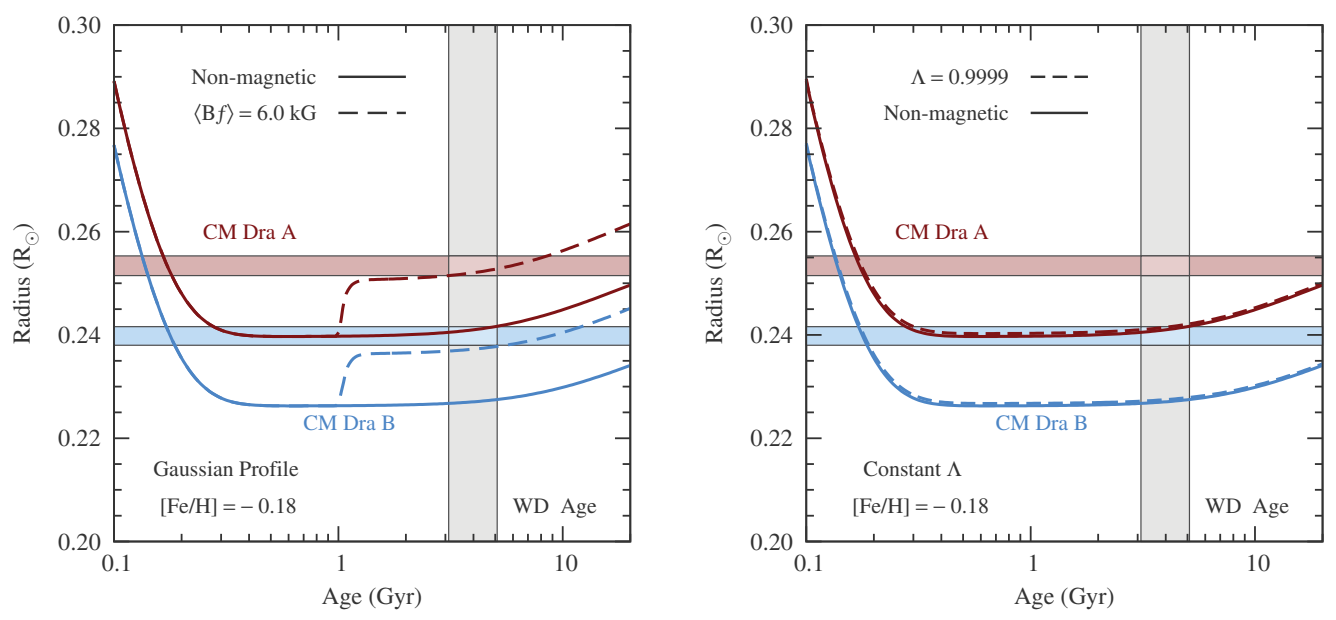

Figure 2. Same as Figure 1, but for the stars of CM Dra. (Left) Models invoking the stabilization of convection initialized at 1 Gyr. (Right) Models that have inhibited convective efficiency initialized at $100 \mathrm{Myr}$.

to correct models of YY Gem to observations. In both cases the peak interior magnetic field strengths are roughly on the order of $1-10 \mathrm{kG}$.

Results are quite different for fully convective stars, as illustrated with a representative system (CM Draconis) in Figure 2. The left panel shows that stabilization of convection is sufficient to reconcile model radii with observations. Models presented in the left panel of Figure 2 have a surface magnetic field strength of $6.0 \mathrm{kG}$ with a peak interior magnetic field strength of roughly $50 \mathrm{MG}$. Inhibiting convective efficiency, however, does not radically alter model predictions. Models in the right panel of Figure 2 have magnetic field strengths equal to $99.99 \%$ of the equipartition value - roughly $3 \mathrm{kG}$ at the surface and $50 \mathrm{kG}$ deep within the star. What is not apparent from Figure 2 is that we adjusted the interior magnetic field strength within the models invoking stabilization of convection to provide greater radius inflation. Fully convective models are sensitive to the deep interior magnetic field strength, which can be set arbitrarily to achieve the desired inflation.

\section{Discussion}

Introducing magneto-convection into stellar models appears to provide at least a qualitative solution to the problem of inflated low-mass stars in DEBs. What must be addressed is whether the magnetic field properties (surface and interior field strengths) are physically realistic. Using a scaling relation between stellar coronal X-ray luminosity and surface magnetic flux (Feiden \& Chaboyer 2013a), we find that models invoking the stabilization of convection require surface magnetic field strengths that are likely too strong. This is particularly evident for partially convective stars where estimated surface magnetic field strengths are too strong by about a factor of 5 . However, models using inhibition of convective efficiency predict surface magnetic field strengths consistent with X-ray luminosity estimates. Assuming a "turbulent dynamo" is primarily responsible for the generation of magnetic fields in low-mass partially convective stars, it appears plausible that magnetic fields are inflating stellar radii.

Surface magnetic field strengths for fully convective stars invoking stabilization of convection appear plausible based on X-ray luminosity estimates. Models using inhibition of convective efficiency, while providing the best agreement with observed surface magnetic 


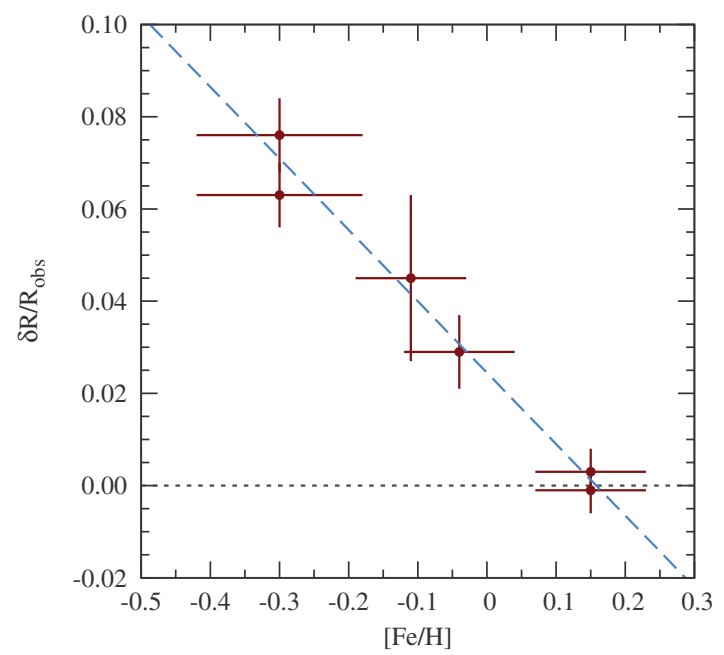

Figure 3. Relative radius error between stellar evolution models and observations of fully convective stars plotted against observationally determined metallicities.

field strengths (Reiners 2012), fail to reproduce observed stellar properties. Recall, we mentioned that the deep interior field strength in fully convective stars was important. Magnetic field strengths must be nearly $50 \mathrm{MG}$ to influence the structure of low-mass fully convective stars. This appears too strong to be physically plausible. A turbulent dynamo mechanism, such as that operating in fully convective stars, cannot produce a magnetic field strength of such magnitude. Instead, a field of that strength must be of primordial origin, which appears inconsistent with estimated diffusion timescales and additional observational evidence (Feiden \& Chaboyer 2013b). Therefore, it seems unlikely, given current evidence, that magnetic fields are responsible for inflating fully convective stars.

Ultimately, we must then view the result for partially convective stars with some skepticism. Further theoretical modeling of magneto-convection and additional observational constraints on properties like metallicity, surface magnetic flux, and star spot coverage are required. Yet, it is not too early to begin searching for other explanations. Among fully convective stars in DEBs, for instance, we find a strong anti-correlation between the level of radius inflation of real stars and estimated stellar metallicities (see Figure 3). At the moment, we have no convincing explanation for this anti-correlation, just that we see it in the DEB data and hints of it in interferometric data (Boyajian et al. 2012).

This work was supported by NSF grant AST-0908345 and the William H. Neukom 1964 Institute for Computational Science at Dartmouth College.

\section{References}

Boyajian, T. S., et al. 2012, ApJ, 757, 112

Feiden, G. A. \& Chaboyer, B. 2012a, ApJ, 757, 42

Feiden, G. A. \& Chaboyer, B. 2012b, ApJ, 761, 30

Feiden, G. A. \& Chaboyer, B. 2013a, arXiv:1309.0033

Feiden, G. A. \& Chaboyer, B. 2013b, in preparation

López-Morales, M. 2007, ApJ, 660, 732

Reiners, A. 2012, Living Reviews in Solar Physics, 8, 1

Ribas, I. 2006, ApESSS, 304, 89 